\title{
Differential regulation of glutamate-cysteine ligase subunit expression and increased holoenzyme formation in response to cysteine deprivation
}

\author{
Jeong-In LEE, Joann KANG and Martha H. STIPANUK ${ }^{1}$ \\ Division of Nutritional Sciences, Cornell University, Ithaca, NY 14853, U.S.A.
}

GCL (glutamate-cysteine ligase) is a heterodimer of a GCLC (GCL catalytic subunit) that possesses all of the enzymatic activity and a GCLM (GCL modifier subunit) that alters the $K_{\mathrm{i}}$ of GCLC for GSH. We hypothesized that the expression of GCLM and the association of GCLM with GCLC were responsible for the apparent increase in GCL activity state observed in the liver of rats fed low-protein diets or in hepatocytes cultured in low-sulphur amino acid-containing medium. Therefore we conducted a series of studies using rats and a human hepatoma (HepG2/C3A) cell line to assess the role of GCLM and holoenzyme formation in the regulation of GCL activity in response to sulphur amino acid intake or availability. Increases in GCL activity in rat liver, as well as in HepG2 cells, were due to the additive effects of changes in the amount of GCLC and the $k_{\text {cat }}$ for GCLC. The increase in the $k_{\text {cat }}$ for GCLC was associated with increased holoenzyme formation, which was associated with an increase in the molar ratio of GCLM to GCLC. Furthermore, our results indicate that the GCLM level in rat liver is always limiting and that up-regulation of the GCLM level results in increased holoenzyme formation and an increase in the $k_{\text {cat }}$. This is the first report demonstrating that the catalytic efficiency of rat GCL is increased by holoenzyme formation and the first demonstration of differential up-regulation of the GCL subunits in response to cysteine deprivation.

Key words: glutamate-cysteine ligase (GCL), glutathione (GSH), cysteine, HepG2/C3A cells, holoenzyme, low-protein diet.

\section{INTRODUCTION}

GSH (glutathione; L- $\gamma$-glutamyl-L-cysteinylglycine) is the major non-protein thiol in mammalian cells. GSH serves as a storage depot for cysteine, allowing maintenance of low cellular cysteine levels while ensuring a supply of cysteine for extrahepatic tissues. GSH reacts non-enzymatically as a reducing or conjugating agent and is involved in a number of enzymatic reactions. These include serving as a cofactor for the glutathione peroxidase family of enzymes and as a cosubstrate for conjugation to a variety of electrophiles by the glutathione S-transferase family of enzymes. As a consequence of these reactions, GSH is either oxidized to GSSG (glutathione disulphide) or is further degraded as part of various conjugates.

The replenishment of GSH may occur via reduction of GSSG by glutathione reductase or by de novo synthesis of GSH from its constituent amino acids. GSH is synthesized by two cytosolic ATP-dependent enzymes: GCL (glutamate-cysteine ligase; also known as $\gamma$-glutamylcysteine synthetase, EC 6.3.2.2) and glutathione synthetase (EC 6.3.2.3). The first step in GSH synthesis, catalysed by GCL, normally regulates the flux of cysteine to GSH. Short-term regulation of GSH production by GCL occurs mainly via the availability of cysteine, the limiting substrate, and by feedback inhibition of GCL by GSH. The depletion of GSH by conjugation and oxidation reactions is generally associated with the synthesis of new GCL subunits as a result of increased gene expression, leading to an increased cellular capacity for GSH synthesis [1-4].

The mammalian GCL holoenzyme consists of a $73 \mathrm{kDa}$ catalytic subunit (GCLC) and a $31 \mathrm{kDa}$ modifier subunit (GCLM), which are encoded by separate genes. It has been shown in vitro that GCLC exhibits catalytic activity and feedback inhibition by GSH, whereas GCLM has no enzymatic activity. However, the association of GCLM with GCLC alters the kinetics of the reaction, lowering the $K_{\mathrm{m}}$ for glutamate and increasing the $K_{\mathrm{i}}$ for GSH [5-7]. Since the initial demonstration that GCL is a heterodimer [8,9] and the characterization of kinetic parameters of the purified enzyme [5,6], it has generally been assumed that the monomer of GCLC is essentially inactive in vivo and that the holoenzyme is the major species present in cells $[10,11]$.

In contrast with the prediction that GCL exists as a heterodimer in vivo, our measurements of GCL kinetics in rat liver homogenates are consistent with the GCLC monomer being the major catalytic species. The $K_{\mathrm{m}}$ for glutamate was $4 \mathrm{mM}$, higher than the reported $K_{\mathrm{m}}$ for rat holoenzyme $(1.4 \mathrm{mM})$ and the $K_{\mathrm{i}}$ for GSH was $1.5 \mathrm{mM}$, lower than the reported $K_{\mathrm{i}}$ for rat holoenzyme $(8.2 \mathrm{mM})$ [5,12]. Also, in contrast with most of the reported studies of GCL regulation in animals, which have only measured the abundance of GCL subunit mRNA, our studies indicated that GCL activity is also regulated at the level of the activity state [13-18]. The increase in GCL activity $\left(V_{\max }\right)$ was greater than the increase in GCLC amount. We hypothesized that the expression of GCLM and the association of GCLM with GCLC is responsible for this observed increase in the activity state.

Therefore we conducted a series of studies using rats and a human hepatoma (HepG2/C3A) cell line to assess the role of GCLM and holoenzyme formation in the regulation of GCL activity in response to sulphur amino acid intake or availability. Our results indicate that GCL activity is regulated both by changes in the amount of GCLC and by changes in the $k_{\text {cat }}$ for the enzyme due to holoenzyme formation. This is the first report demonstrating that the catalytic efficiency of rat GCL is increased by

Abbreviations used: DCF, 2',7'-dichlorofluorescein; DCFH-DA, 2',7'-dichlorodihydrofluorescein diacetate; DMEM, Dulbecco's modified Eagle's medium; DTT, dithiothreitol; GCL, glutamate-cysteine ligase; GCLC, GCL catalytic subunit; GCLM, GCL modifier subunit; HBE1, human bronchial epithelial 1; ROS, reactive oxygen species; TBARS, thiobarbituric acid reactive substances.

1 To whom correspondence should be addressed (email mhs6@cornell.edu). 
holoenzyme formation and the first demonstration of differential up-regulation of the GCL subunits in response to cysteine deprivation.

\section{EXPERIMENTAL}

\section{Expression and purification of GCLM and GCLC}

The cDNA for GCLM was obtained by PCR using total human RNA and the following primers: forward, 5'-TAGGATCCATGGGCACCGACAGCCGCGCGGCCAAG-3' and reverse, 5' TTAAGCTTAGAACCCCTTCTTTTAGCTTG- $3^{\prime}$. The cDNA for GCLC was similarly obtained using the following primers: forward, 5'-TTGGCATGCATGGGGCTGTCCCAGGGCTC-3' and reverse, 5'-CTGGGTACCCTAGTTGGATGAGTCAGTTTT$3^{\prime}$. The resulting DNAs were ligated into the pQE30 plasmid after digestion by HindIII/BamHI for GCLM and SpnI/KpnI for GCLC. The sequence and orientation of the constructs, which included an $\mathrm{N}$-terminal $\mathrm{His}_{6}$ tag, were verified at the BioResource Center (Cornell University, Ithaca, NY, U.S.A.).

Escherichia coli M15 were transformed with pQE30-GCLC or pQE30-GCLM, and $\mathrm{His}_{6}$-GCLC or $\mathrm{His}_{6}$-GCLM was expressed by growing transformed cells to an $A_{600}$ of 0.7 at $37^{\circ} \mathrm{C}$, cooling the cell suspension and then inducing expression by addition of $1 \mathrm{mM}$ isopropyl $\beta$-D-thiogalactoside with shaking overnight at room temperature. Bacteria were harvested in binding buffer [5 mM imidazole, $0.5 \mathrm{M} \mathrm{NaCl}$ and $20 \mathrm{mM}$ Tris/HCl $(\mathrm{pH} 7.9)$ ] containing Igepal CA-630, $0.1 \%$ Nonidet P40 and protease inhibitors. The bacterial suspension was sonicated and centrifuged at $20000 \mathrm{~g}$ for $30 \mathrm{~s}$ and the supernatant was loaded on to an $\mathrm{Ni}^{2+}$. nitrilotriacetate column (Novagen, Darmstadt, Germany) and eluted using imidazole. Fractions containing GCLC or GCLM were pooled, concentrated and exchanged for $20 \mathrm{mM}$ Tris buffer with $150 \mathrm{mM} \mathrm{NaCl}, 1 \mathrm{mM}$ DTT (dithiothreitol) and $10 \%$ (v/v) glycerol $(\mathrm{pH} 7)$ by three sequential concentration steps. The purified proteins were stored at $-70^{\circ} \mathrm{C}$.

\section{Antibodies}

Rabbit anti-GCLC serum was a gift from Dr Henry J. Forman (University of California, Merced, CA, U.S.A.). The specificity of this anti-GCLC antibody was described previously [13]. Rabbit anti-GCLM serum was generated by Proteintech Group, Inc. (Chicago, IL, U.S.A.) using purified recombinant human GCLM as the antigen. Anti-GCLM antibody was purified from serum using an Affi-Gel Protein A column and kit (Bio-Rad).

\section{Animals and dietary treatments}

Male Sprague-Dawley rats that weighed approx. 170-210 g were purchased from Harlan Sprague Dawley (Indianapolis, IN, U.S.A.). Rats were fed semi-purified diets that contained a high level of protein ( $400 \mathrm{~g}$ of casein $/ \mathrm{kg}$ ) for 2 weeks and then switched to a diet with a low level of protein (100 $\mathrm{g}$ of casein $/ \mathrm{kg}$ ). Housing conditions, feeding protocol and diet composition were the same as reported for a previous study [16]. Rats that had been randomly assigned to time points $(n=3)$ were killed at $0,12,24,48,72$ or $144 \mathrm{~h}$ after the start of the new diet. Rats were anaesthetized with $\mathrm{CO}_{2}$ and decapitated. Livers were removed, rinsed with icecold saline, frozen in liquid nitrogen and stored at $-140^{\circ} \mathrm{C}$ until later analysis. For GCL activity assay and Western blot analysis, frozen liver was homogenized in $0.05 \mathrm{M}$ phosphate buffer (pH 6.8) and centrifuged at $20000 \mathrm{~g}$ to obtain the supernatant. For Northern-blotting, frozen liver was homogenized in denaturing solution and total RNA was isolated. The experimental protocol was approved by the Cornell University Institutional Animal Care and Use Committee.

\section{Cultured HepG2/C3A cells}

HepG2/C3A cells were cultured in a humidified incubator at $37^{\circ} \mathrm{C}$ and $5 \% \mathrm{CO}_{2}$ in sulphur amino acid-free DMEM (Dulbecco's modified Eagle's medium) supplemented with $10 \%$ (v/v) foetal bovine serum, $2 \mathrm{mM}$ L-glutamate, $1 \mathrm{mM}$ sodium pyruvate, $0.1 \mathrm{mM}$ L-methionine, $1 \times$ MEM non-essential amino acid solution, $0.05 \mathrm{mM}$ bathocuproine disulphonate and the indicated amount of cysteine or methionine added. Cells were plated at a density of a $1 \times 10^{6}$ cells $/ 100 \mathrm{~mm}$ diameter culture dish in 'standard' medium ( $+0.1 \mathrm{mM}$ cysteine). On the following day, medium was replaced with experimental medium and cells were cultured for 24 or $36 \mathrm{~h}$; for cells harvested at $36 \mathrm{~h}$, the medium was replaced with fresh medium at $24 \mathrm{~h}$. Cells were washed with icecold PBS and then collected in PBS for analysis of cysteine, GSH, DCF $\left(2^{\prime}, 7^{\prime}\right.$-dichlorofluorescein) and TBARS (thiobarbituric acid reactive substances); in $50 \mathrm{mM}$ Tris/ $\mathrm{HCl}(\mathrm{pH} 8)$ for GCL activity assay; in lysis buffer for Western blot analyses; or in denaturation solution for Northern blot analysis.

\section{GCL activity assay}

GCL activity was analysed under conditions approaching $V_{\max }$ as described by Yan and Huxtable [19] using $50 \mathrm{mM}$ L-glutamate and $5 \mathrm{mM}$ L-cysteine as substrates. $\gamma$-Glutamylcysteine was quantified by HPLC according to the method of Fariss and Reed [20] as modified by Stipanuk et al. [21]. The concentration of soluble protein in the cell lysates was determined according to the method of Smith et al. [22].

\section{Western blot analysis}

GCLC and GCLM, as well as holoenzyme, were measured by Western-blotting of soluble proteins as described previously $[13,17]$. Standard curves were generated by loading incremental amounts of purified human GCLC and GCLM on to each gel. Amounts of supernatant containing $100 \mu \mathrm{g}$ of total soluble proteins were separated by one-dimensional SDS/PAGE using 10 or $7.5 \%(\mathrm{w} / \mathrm{v})$ gels. SDS/PAGE was run under both reducing and non-reducing conditions. For the reducing conditions, equal amounts of total soluble protein were mixed with loading buffer that contained $80 \mathrm{mM}$ DTT, incubated at $95^{\circ} \mathrm{C}$ for $4 \mathrm{~min}$ and then loaded on to the gels. For non-reducing conditions, protein was not treated with DTT or heat before loading. Native PAGE was performed using $7.5 \%(\mathrm{w} / \mathrm{v})$ gels run in the absence of DTT.

\section{Northern blot analysis}

GCLC and GCLM mRNA levels were measured by quantitative Northern blot analysis as described previously [13,17]. cDNA probes corresponding to bp 397-863 of GCLC were synthesized using RT (reverse transcription)-PCR. The sequence of the probes was verified at the BioResource Center. Radiolabelled cDNA probes were prepared using $\left[{ }^{32} \mathrm{P}\right] \mathrm{dCTP}$ and random priming.

\section{Cysteine and glutathione levels}

Total cysteine and glutathione (GSH and GSSG forms) in cells were measured by formation of $S$-carboxymethyl derivatives followed by chromophore derivatization of primary amines with 1-fluoro-2,4-dinitrobenzene and separation of these derivatives by reversed-phase ion-exchange HPLC using a 3-aminopropyl column (Brownlee Labs, Santa Clara, CA, U.S.A.) as described previously [22]. 


\section{TBARS assay}

TBARS were measured as described by Kikugawa et al. [23] using tetraethoxypropane [malondialdehyde-bis-(diethyl-acetal)] as the standard.

\section{DCF production assay}

To assay intracellular ROS (reactive oxygen species), cells were treated with non-fluorescent DCFH-DA $\left(2^{\prime}, 7^{\prime}\right.$-dichlorodihydrofluorescein diacetate) (Molecular Probes, Eugene, OR, U.S.A.). Immediately before use, DCFH-DA was dissolved in $100 \%$ DMEM to give a $2.5 \mathrm{mM}$ solution that was then diluted to $10 \mu \mathrm{M}$ with PBS. Cell culture medium was discarded and $5 \mathrm{ml}$ of the $10 \mu \mathrm{M}$ DCFH-DA/PBS solution was added to $35 \mathrm{~mm}$ cell culture dishes and cells were incubated for $30 \mathrm{~min}$ at $37^{\circ} \mathrm{C}$. A sample of the extracellular solution was collected to measure the amount of DCF released from the cells. Cells were then washed with PBS, harvested into $1 \mathrm{ml}$ of fresh PBS, sonicated and the supernatant was used to measure intracellular DCF. DCF fluorescence was measured using an excitation wavelength of $485 \mathrm{~nm}$ and an emission wavelength of $535 \mathrm{~nm}$.

\section{Statistical analysis}

Results were statistically analysed by a one-way ANOVA and Tukey's $\omega$-procedure or Dunnett's comparison, for comparison of mean values (Minitab, Release 13.1).

\section{RESULTS}

Rat hepatic GCL activity, and GCLC and GCLM levels are up-regulated in response to a low-protein diet

Rats were first adapted to a high-protein diet (400 g of casein $/ \mathrm{kg}$ ) over a 2 -week period and then switched to a low-protein diet $(100 \mathrm{~g}$ of casein $/ \mathrm{kg})$. Hepatic GCL activity was low in rats that were adapted to the high-protein diet and increased gradually over the 6-day period after introduction of the low-protein diet (Figure 1A). Quantitative measurement of the molar amounts of GCLC and GCLM in liver demonstrated that the amount of both subunits increased, reaching peak levels by day 3 , but that the increase in GCLM was greater than that of GCLC (Figures 1B and 1C). By day 6 after the change to the low-protein diet, the amount of GCLC and GCLM was 1.3 times and 1.8 times the initial levels respectively, whereas GCL activity as assayed under $V_{\max }$ conditions was 2.9 times the initial level. The greater increase in GCL activity than in GCLC level indicated that the $k_{\text {cat }}$ for GCL had increased.

\section{The GCLM/GCLC molar ratio and the $\boldsymbol{k}_{\text {cat }}$ of GCL are increased in the livers of rats fed on a low-protein diet}

The molar ratio of GCLM/GCLC in the liver of rats fed the highprotein diet was 0.23 and this increased to $0.34(1.5 \times$ initial value) following the switch to a low-protein diet (Figure 2A). The relatively low abundance of GCLM in the liver of rats is consistent with our previous observation that the kinetics for GCL in rat liver homogenates resembled those of the GCLC monomer [12]. Nevertheless, the relative increase in the GCLM/GCLC ratio in rats that were switched to a low-protein diet suggests that increased holoenzyme formation may contribute to the higher total GCL activity observed in the liver of rats fed on low-protein diets.

We calculated the $k_{\text {cat }}$ values for liver GCLC. The $k_{\text {cat }}$ for GCL from the liver of rats fed on the high-protein diet was $100 \pm$ $13 \mathrm{nmol}$ of product/min per nmol of GCLC (mean \pm S.E.M.) and increased up to $222 \pm 16 \mathrm{nmol}$ of product/min per nmol of
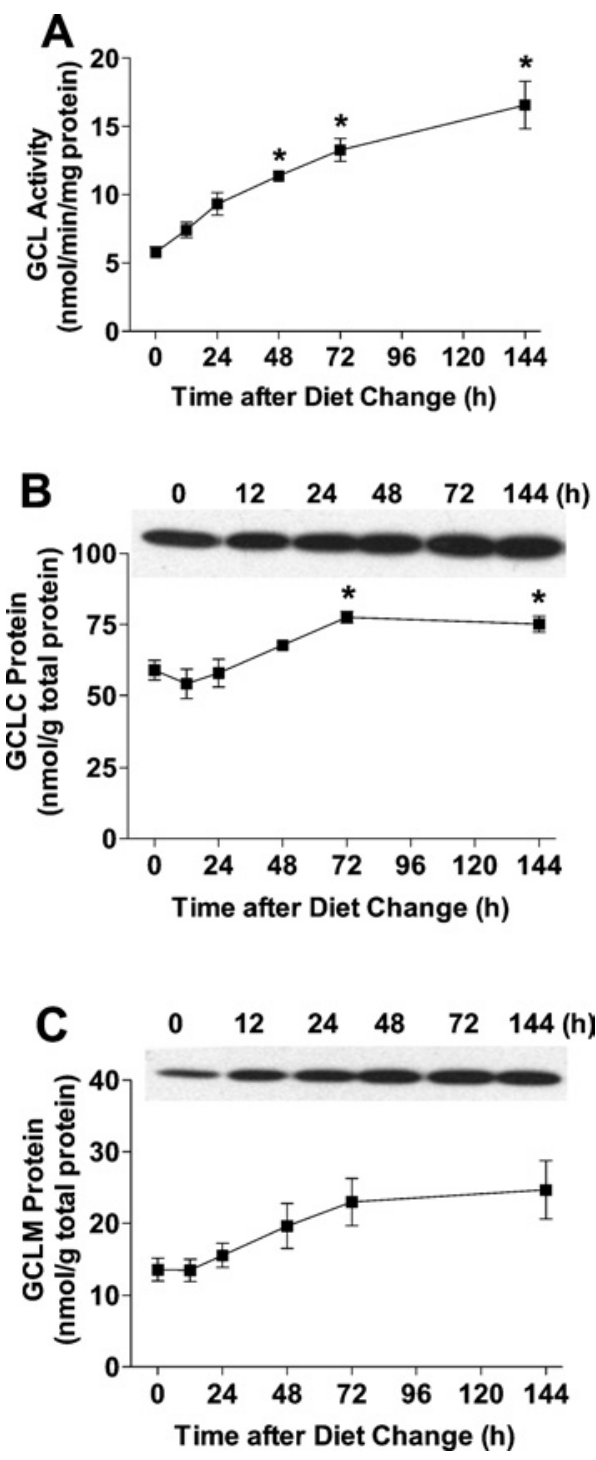

Figure 1 Rat hepatic GCL activity, and GCLC and GCLM levels are upregulated in response to a low-protein diet

(A) GCL activity in the liver of rats adapted to a high-protein ( $400 \mathrm{~g}$ of casein $/ \mathrm{kg}$ ) diet and switched to a low-protein (100 g of casein/ $\mathrm{kg}$ ) diet at zero time. GCLC protein (B) and GCLM protein $(\mathbf{C})$ in the liver of rats adapted to a high-protein diet and switched to a low-protein diet at zero time. A representative Western blot is shown for each protein; $100 \mu \mathrm{g}$ of total soluble protein was loaded in each lane. The amounts of GCLC and GCLM were calculated using a standard curve run on each gel by loading various amounts of purified GCLC or GCLM protein. Values denoted by * are significantly different $(P \leqslant 0.05)$ than the initial 0 time value as determined by ANOVA and Dunnett's procedure. Each value is the mean \pm S.E.M. for 3 rats.

GCLC after the rats were placed on the low-protein diet (Figure $2 \mathrm{~B}$ ). The change in $k_{\text {cat }}$ occurred in a time-dependent manner over the 6-day period, consistent with the time-dependent increase in the GCLM/GCLC molar ratio (Figure 2A). The 30\% increase in the absolute level of GCLC, corrected for the $120 \%$ higher $k_{\text {cat }}(1.3 \times 2.2=2.9)$, completely accounts for the $190 \%$ higher GCL activity level in the liver of rats adapted to the low-protein diet than in the liver of rats adapted to the highprotein diet.

\section{GCLM increases the GCL activity via holoenzyme formation in vitro}

The amount of GCLM in rat liver is clearly insufficient to convert all GCLC to holoenzyme. To further test the hypothesis that 

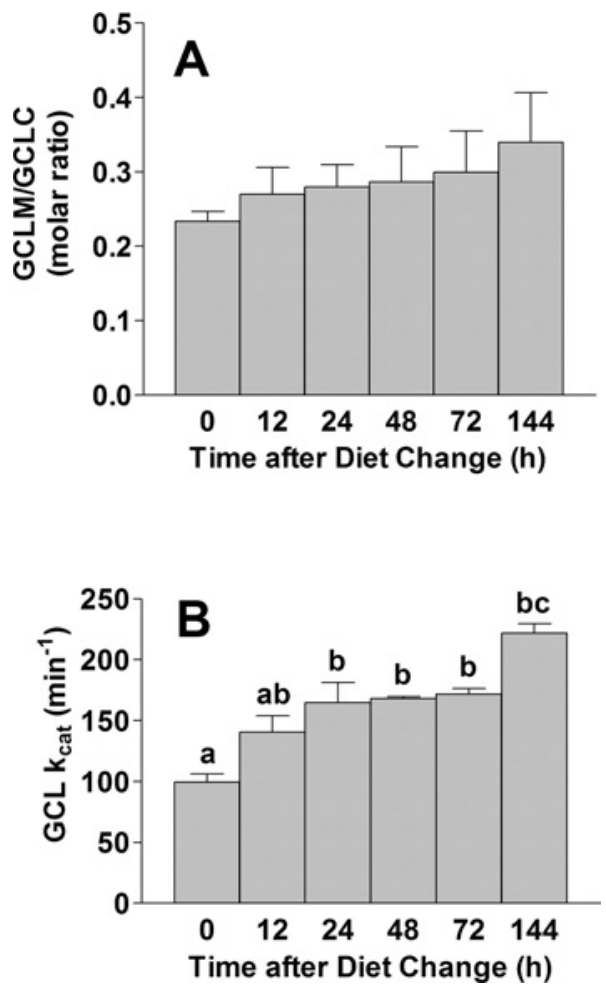

Figure 2 The GCLM/GCLC molar ratio and $\boldsymbol{k}_{\text {cat }}$ of GCL are increased in liver of rats fed a low-protein diet

(A) The GCLM/GCLC molar ratio in the liver of rats fed on a high-protein diet and switched to a low-protein diet at 0 time. The molar concentrations of GCLC and GCLM were calculated by dividing the amount of GCLC (mg) or GCLM (mg) by the molecular mass of the protein; the molar amounts were used to calculate the ratio. (B) The $k_{\text {cat }}$ values of whole liver GCL at each time point. The $k_{\text {cat }}$ was calculated by dividing the $V_{\max }$ (mol of product formed per min) by the molar amount of GCLC. Bars not denoted by the same letter are significantly different $(P \leqslant 0.05)$ as determined by ANOVA and Tukey's $\omega$-procedure. Each value is the mean \pm S.E.M. for 3 rats.

GCLM increases GCL activity via holoenzyme formation, we tested the effect of addition of recombinant GCLM to the homogenates of liver from rats fed on the high-protein diet. Addition of GCLM increased GCL activity in a dose-dependent manner as shown in Figure 3(A). In addition, analysis of the homogenates by native gel electrophoresis indicated that the addition of GCLM progressively shifted the GCLC to holoenzyme as the GCLM/GCLC ratio was increased from 0.2 to 3.0 (Figures 3B and $3 \mathrm{C}$ ). Note that little GCLC was present as holoenzyme in the homogenate to which no purified GCLM was added, consistent with the kinetic parameters we have routinely observed for rat liver homogenates, whereas approx. $80 \%$ of the total GCLC was present as holoenzyme in the homogenate to which GCLM had been added to increase the GCLM concentration to $2.2 \mu \mathrm{M}$ and the GCLM/GCLC molar ratio to 3.0. Thus GCL activity increased by approx. $40 \%$ as the fixed amount of GCLC was shifted from mainly monomer to mainly holoenzyme (Figure 3).

\section{Effects of sulphur amino acid level in the culture medium on intracellular cysteine and GSH levels in HepG2/C3A cells}

The increase in GCL activity after addition of excess GCLM in the in vitro study was less than that observed in the liver of rats switched from a high-protein to a low-protein diet, despite the observation that essentially all the GCLC was converted to holoenzyme in the in vitro study whereas the concentration of GCLM in rat liver remained limiting for holoenzyme formation.
A

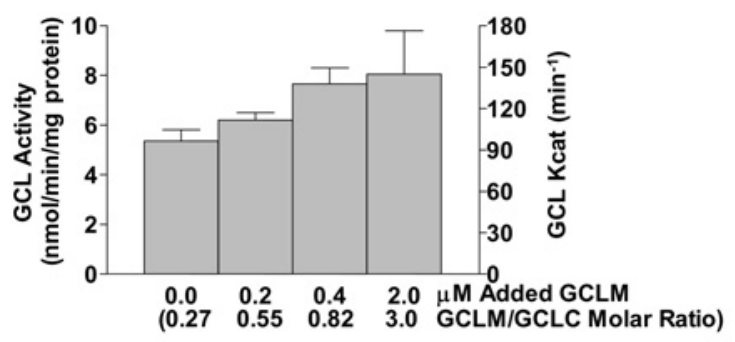

B $\begin{array}{llll}0 & 0.2 & 0.4 & 2.0 \mu \mathrm{M} \text { Added GCLM }\end{array}$

(l) $0.270 .550 .82 \quad 3.0$ GCLM/GCLC Molar Ratio)

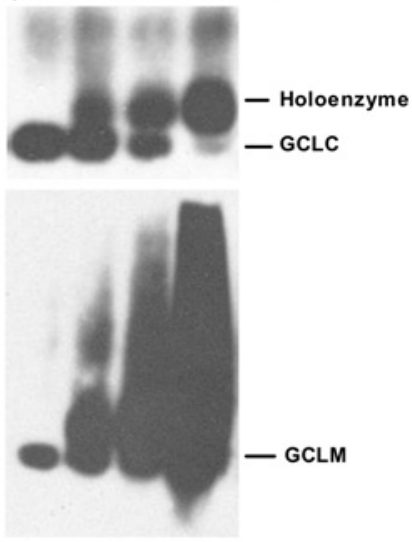

C

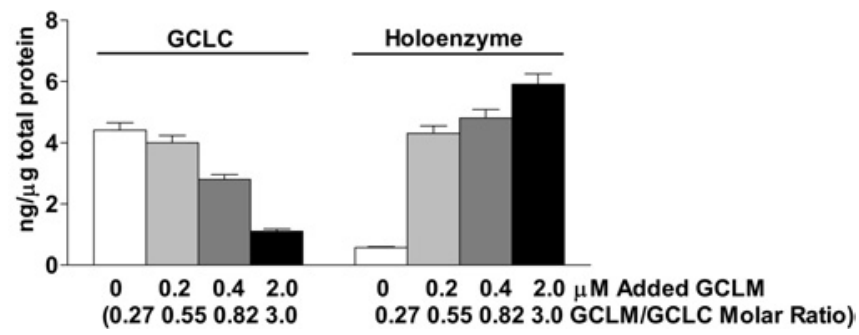

Figure 3 GCLM increases GCL activity via holoenzyme formation in vitro

(A) The GCL activities and GCLC $k_{\text {cat }}$ values for liver homogenate from rats fed on a highprotein diet and incubated with incremental amounts of recombinant human GCLM for $30 \mathrm{~min}$ before enzyme assay at $37^{\circ} \mathrm{C}$. The final concentrations of the added recombinant GCLM are shown along the $x$-axis together with the GCLM/GCLC molar ratio calculated as the sum of the (rat GCLM + recombinant GCLM) divided by the rat GCLC present in the homogenate. Each value is the mean \pm S.E.M. for 3 rats. (B) Liver homogenate treated as described for $(\mathbf{A})$ was subjected to native gel electrophoresis under non-reducing conditions (no DTT and no heat) for $30 \mathrm{~min} ; 100 \mu \mathrm{g}$ of total soluble protein was loaded on to each lane. No SDS was included in the sample buffer or gel. After electrophoresis and transfer, the membrane was incubated with anti-GCLC (upper blot) for GCLC and holoenzyme detection. The membrane was stripped and GCLM was detected using anti-GCLM (lower blot). (C) GCLC and holoenzyme detected in (B) were quantified using standard curves generated using recombinant GCLC protein.

To further explore the role of holoenzyme formation in the increase in GCL activity under conditions of limiting sulphur amino acids, we performed a series of studies using HepG2/C3A cells. In order to establish the sulphur amino acid levels to be used in the medium in these studies, we first tested the effect of the addition of cysteine or methionine to a basal medium that contained $0.1 \mathrm{mM}$ methionine. Intracellular concentrations of reduced cysteine and GSH in the HepG2/C3A cells are shown in Table 1.

Intracellular cysteine and GSH levels increased in a step-wise fashion with increases in cysteine in the medium. Cysteine, but not GSH, levels were lower at $24 \mathrm{~h}$, just before the medium was changed, than they were at $36 \mathrm{~h}$ (i.e. $12 \mathrm{~h}$ after the medium had 
Table 1 Free intracellular reduced cysteine and glutathione in cultured HepG2/C3A cells after 24 and $36 \mathrm{~h}$ of culture in basal medium supplemented with various amounts of cysteine or methionine

The basal medium contained $0.05 \mathrm{mM}$ foetal calf serum and $0.1 \mathrm{mM} \mathrm{L-methionine;} \mathrm{the} \mathrm{additions}$ listed are supplements to the basal medium. ND (not detected) means the value is $\leqslant 0.2 \mathrm{nmol}$ of cysteine/mg of soluble protein. Medium was changed every $24 \mathrm{~h}$, so the $36 \mathrm{~h}$ values reflect concentrations $12 \mathrm{~h}$ after the medium change and the $24 \mathrm{~h}$ values reflect concentrations $24 \mathrm{~h}$ after the last medium change. Values are the average of two experiments or the mean + S.E.M. for 3 separate experiments.

\begin{tabular}{|c|c|c|c|c|}
\hline \multirow{2}{*}{$\begin{array}{l}\text { Sulphur amino acid } \\
\text { supplement (mM) }\end{array}$} & \multicolumn{2}{|c|}{$\begin{array}{l}\text { Cysteine } \\
\text { (nmol/mg of soluble protein) }\end{array}$} & \multicolumn{2}{|c|}{$\begin{array}{l}\text { GSH } \\
\text { (nmol/mg of soluble protein) }\end{array}$} \\
\hline & $24 \mathrm{~h}$ & $36 \mathrm{~h}$ & $24 \mathrm{~h}$ & $36 \mathrm{~h}$ \\
\hline $\begin{array}{l}\text { Basal medium } \\
\text { Cysteine }\end{array}$ & ND & ND & 6.7 & $4.2 \pm 1.1$ \\
\hline 0.05 & 0.3 & $0.5 \pm 0.7$ & 16 & $22 \pm 11$ \\
\hline 0.1 & $0.6 \pm 0.7$ & $2.2 \pm 0.7$ & 27 & $42 \pm 26$ \\
\hline 0.3 & 1.5 & $7.7 \pm 4.8$ & 34 & $44 \pm 13$ \\
\hline 0.5 & $7.8 \pm 4.5$ & $15.4 \pm 1.8$ & 82 & $60 \pm 9$ \\
\hline \multicolumn{5}{|l|}{ Methionine } \\
\hline 0.05 & ND & ND & 7.0 & 4.1 \\
\hline 0.1 & ND & ND & 7.3 & 4.3 \\
\hline 0.3 & ND & ND & 7.0 & 4.2 \\
\hline 0.5 & ND & ND & 6.2 & 3.7 \\
\hline
\end{tabular}

been replaced with fresh culture medium). It is noteworthy that the addition of methionine had no effect on intracellular cysteine or GSH concentrations at either 24 or $36 \mathrm{~h}$. HepG2/C3A cells lack the high- $K_{\mathrm{m}}$ form of methionine adenosyltransferase and clearly have a limited capacity to convert methionine sulphur to cysteine $[23,24]$. Our results clearly demonstrate that these cells require cysteine in the medium for optimal GSH production. Cysteine was not detectable in the HepG2/C3A cells, and the level of GSSG was usually less than $0.5 \mathrm{nmol} / \mathrm{mg}$ of soluble protein and always less than $3 \mathrm{nmol} / \mathrm{mg}$ of soluble protein. Export of GSSG into the medium was not observed in any of the conditions listed in Table 1, although GSSG export was observed at very high cysteine concentrations (1.5 and $2.5 \mathrm{mM}$ cysteine in the culture medium; results not shown).

\section{Effect of sulphur amino acid level in culture medium on the level of ROS in HepG2/C3A cells}

To determine whether changes in the cysteine and GSH levels were associated with oxidative stress, which would result in activation of GCLC and GCLM gene transcription, we evaluated the presence of ROS by measurement of DCFH oxidation and TBARS formation. As shown in Figure 4, measurement of DCF production indicated that ROS generation in HepG2/C3A cells was not affected by variations in the cysteine or methionine concentration in the medium $(P>0.05)$ despite the effects of cysteine supplementation on intracellular levels of both cysteine and GSH. Similar results were observed using the TBARS assay (results not shown). The ability to manipulate cellular cysteine and GSH levels without causing oxidative stress allows for the study of the effect of cysteine availability on GCL, independently of the effects of oxidative stress on GCL.

\section{Effects of sulphur amino acid level in culture medium on the levels of GCLC and GCLM mRNAs, and GCL activity, in HepG2/C3A cells}

The amounts of GCLC and GCLM mRNAs were measured in cells cultured with various levels of sulphur amino acids for $36 \mathrm{~h}$ (Figures 5A and 5B). Two distinct transcripts (GCLC, 3.2 and $4.1 \mathrm{~kb}$; GCLM, 1.4 and $4.1 \mathrm{~kb}$ ) were observed for each subunit

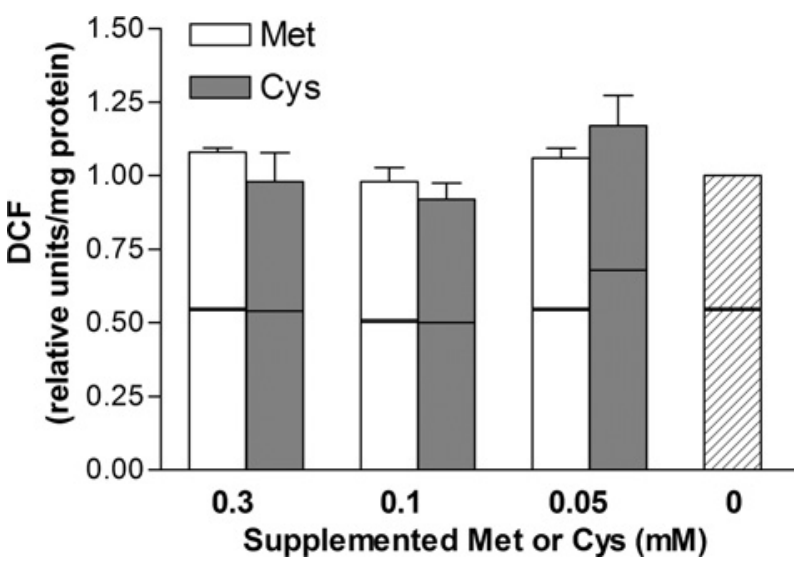

Figure 4 Effect of sulphur amino acid level in the culture medium on ROS formation in HepG2/C3A cells

Relative values for intra- and extra-cellular DCF formation in HepG2/C3A cells that had been cultured in medium containing $0.1 \mathrm{mM}$ methionine and the designated concentration of supplemental cysteine or methionine for $36 \mathrm{~h}$. Upper box represents intracellular DCF and bottom box represents DCF released from cells into medium. DCF fluorescence was set at 1.0 for the total DCF fluorescence in cell cultured in cysteine-depleted medium ( $0.1 \mathrm{mM}$ methionine). Each value is the mean \pm S.E.M. for three replicates.

mRNA, consistent with the documented alternative processing of GCLC and GCLM mRNAs [26]. Addition of methionine had relatively little effect on the mRNA levels for GCLC and GCLM except at the highest concentration of supplemented methionine. By contrast, addition of even the smallest amount of supplemented cysteine $(0.05 \mathrm{mM})$ markedly decreased the level of both GCLC and GCLM mRNAs to basal levels that were not further reduced by the addition of higher levels of cysteine to the medium. In general, the changes in GCL mRNA level paralleled the changes in intracellular cysteine or GSH concentration, despite no change in the level of ROS. An increase in GCLM mRNA at an intracellular cysteine level of $<0.5 \mathrm{nmol} / \mathrm{mg}$ of soluble protein (medium concentration, $<0.05 \mathrm{mM}$ ) indicates a possible role for intracellular cysteine and GSH, independently of oxidative stress, in regulating GCL subunit expression.

GCL activity in lysates of the HepG2 cells did not parallel the changes in GCLC or GCLM mRNAs (Figures 5A and 5B) but increased in a dose-dependent manner with decreases in cysteine concentration over the range $0.3-0 \mathrm{mM}$ (Figure $5 \mathrm{C}$ ). Thus, in contrast with our previous observations in rat liver and rat hepatocytes $[15,17]$, changes in the level of GCL activity in HepG2 cells did not closely parallel changes in GCL subunit mRNA levels.

\section{Both GCLC and GCLM levels are up-regulated in response to cysteine deprivation in HepG2/C3A cells}

Because we were able to modify the intracellular cysteine concentrations of $\mathrm{HepG} 2 / \mathrm{C} 3 \mathrm{~A}$ cells by varying the concentration of cysteine in the medium and to obtain a range of cysteine levels (0.05-0.3 mM) at which GCLM and GCLC mRNA levels did not appear to be regulated via oxidative stress signalling pathways, we used HepG2/C3A cells to conduct further studies on the regulation of GCL activity via changes in the GCLM/GCLC ratio in response to cysteine availability. As shown in Figure 5(C), the effect of cysteine depletion on GCL activity appeared to occur in a doseresponsive manner, with GCL activity increasing from $1.2 \pm 0.5$ ( $0.3 \mathrm{mM}$ cysteine) to $2.5 \pm 0.7$ (no cysteine) (mean \pm S.E.M.) $\mathrm{nmol} / \mathrm{min}$ per mg of protein as measured after $36 \mathrm{~h}$ of culture in the various media. Addition of higher levels of cysteine did not result 


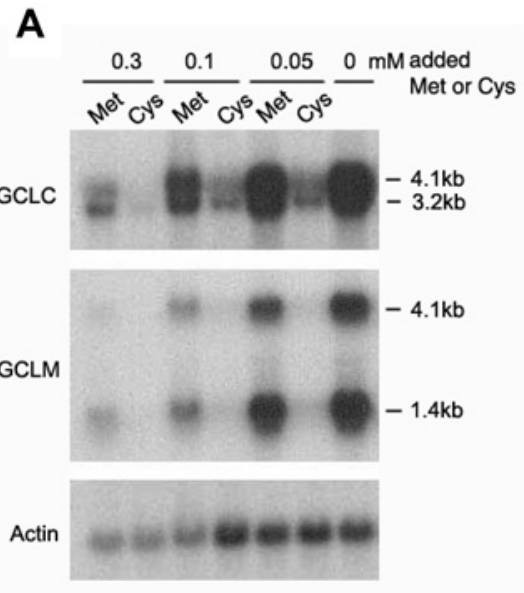

B
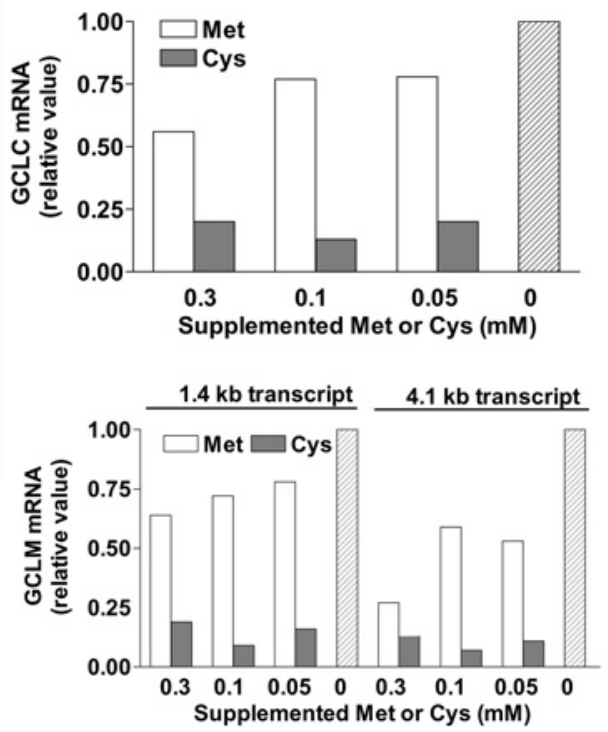

C

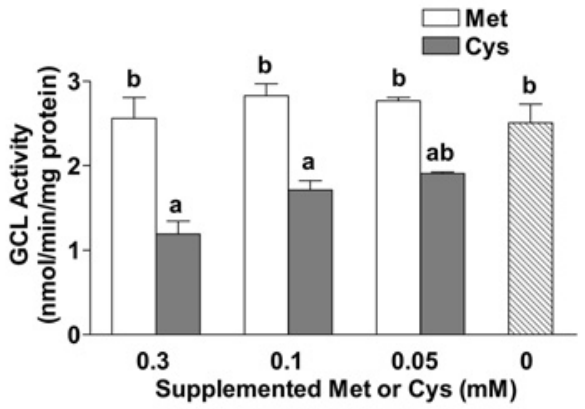

Figure 5 Effects of sulphur amino level in the culture medium on GCLC and GCLM mRNA levels and GCL activity in HepG2/C3A cells

(A) A representative Northern blot of mRNA levels for GCLC and GCLM in HepG2/C3A cells cultured for $36 \mathrm{~h}$ in medium containing $0.1 \mathrm{mM}$ methionine and the designated concentration of supplemental cysteine or methionine. Actin mRNA was probed as an internal standard. Equal amounts of total RNA from three samples were pooled for a given group. (B) The relative amounts of GCLC or GCLM mRNAs shown in (A) were calculated. The two transcripts for GCLC were quantified together and the 4.1 and $1.4 \mathrm{~kb}$ transcripts for GCLM were quantified separately. The relative amount of mRNA for each enzyme sample was calculated using a standard curve of integrated density units versus the amount of total RNA loaded and the amount was divided by the total amount of RNA $(\mu \mathrm{g})$ loaded. Actin mRNA was used to adjust values as a control for variations in loading and transfer. The relative value was set at 1.0 for mRNAs in cells cultured in unsupplemented medium (containing only $0.1 \mathrm{mM}$ methionine). (C) GCL activity in HepG2/C3A cells cultured for $36 \mathrm{~h}$ with various amounts of supplemental methionine or cysteine. Bars not denoted by the same letter are significantly different $(P \leqslant 0.05)$ as determined by ANOVA and Tukey's $\omega$-procedure. Each value is the mean \pm S.E.M. for three replicates.

in lower GCL activity than observed in cells supplemented with $0.3 \mathrm{mM}$ cysteine; in fact, levels of cysteine above $0.5 \mathrm{mM}$ caused up-regulation of GCL, presumably in response to the oxidative stress caused by the high levels of cysteine (results not shown).

Both GCLC and GCLM levels tended to increase with cysteine depletion. The GCLC and GCLM levels were 34 and $84 \%$ higher respectively in cells cultured in cysteine-free medium than in cells cultured in medium supplemented with $0.3 \mathrm{mM}$ cysteine (Figures 6A and 6B). The increase in GCLC and GCLM levels clearly occurred in a step-wise fashion in response to cysteine depletion over the range $0.3-0 \mathrm{mM}$ cysteine, but the mRNA levels for GCLC and GCLM were not up-regulated except when the medium contained no cysteine (Figures 5A and 5B), suggesting that post-transcriptional regulation is involved in the step-wise response of GCLM and, perhaps, GCLC in HepG2 cells.

\section{The GCLM/GCLC molar ratio is increased in HepG2/C3A cells cultured in cysteine-deficient medium}

Although the molar ratio of GCLM to GCLC in HepG2/C3A cells was much higher than in rat liver, the GCLM/GCLC ratio was still sensitive to cysteine depletion. The greater increase in the amount of GCLM than in that of GCLC in response to cysteine deprivation resulted in an increase in the molar ratio in a step-wise fashion from 1.5 to 2.1 , a $40 \%$ increase (Figure $6 \mathrm{C}$ ).

\section{An increase in the GCLM/GCLC molar ratio is paralled by increases in the $\boldsymbol{k}_{\text {cat }}$ of GCL and in the apparent strength of association of subunits in the holoenzyme}

The $k_{\text {cat }}$ for GCL in HepG2/C3A cells changed with progressive cysteine deprivation, increasing in a step-wise manner from $413 \mathrm{nmol}$ of product/min per nmol of GCLC in cells cultured in medium with $0.3 \mathrm{mM}$ cysteine to $657 \mathrm{nmol}$ of product/min per nmol of GCLC in cells cultured without cysteine (Figure 6D). In the case of rat liver, the $110 \%$ increase in GCL activity in HepG2/ $\mathrm{C} 3 \mathrm{~A}$ cells can be accounted for by the $60 \%$ increase in the amount of GCLC and the $30 \%$ increase in the $k_{\text {cat }}$ of GCL: 1.3 GCLC $\times 1.6$ $k_{\text {cat }}=2.1$ (GCL activity).

Native gel electrophoresis indicated that GCL existed primarily as a holoenzyme in HepG2/C3A cells (Figure 7A). This is consistent with the high molar ratios of GCLM/GCLC, with GCLM present in excess of GCLC. With non-reducing SDS/PAGE, GCLC and holoenzyme were partially resolved, with a relatively greater portion of the total GCLC resolving as a monomer in samples from cells cultured in $0.3 \mathrm{mM}$ cysteine and relatively more resolving as the holoenzyme in samples from cells cultured in the absence of cysteine (Figure 7B, left panel). When samples were treated with $80 \mathrm{mM}$ DTT and heat before separation by SDS/PAGE, the holoenzyme entirely disappeared in samples from cells cultured in $0.1 \mathrm{mM}$ cysteine but not in samples from cells cultured without cysteine (Figure 7B, right panel). Thus a portion of holoenzyme in the most cysteine-deficient cells appeared to be resistant to reduction and denaturation.

\section{DISCUSSION}

\section{Regulation of GCL activity occurs by changes in GCLC level and by an increase in its activity state $\left(k_{\text {cat }}\right)$}

Rat and human GCLs are very similar: the amino acid sequence of human GCLC is $94 \%$ identical (97\% similar) and that of human GCLM is $92 \%$ identical ( $94 \%$ similar) to the rat homologues. Thus we might expect rat and human GCL to be regulated in similar manners, as was observed in these studies using intact 
A
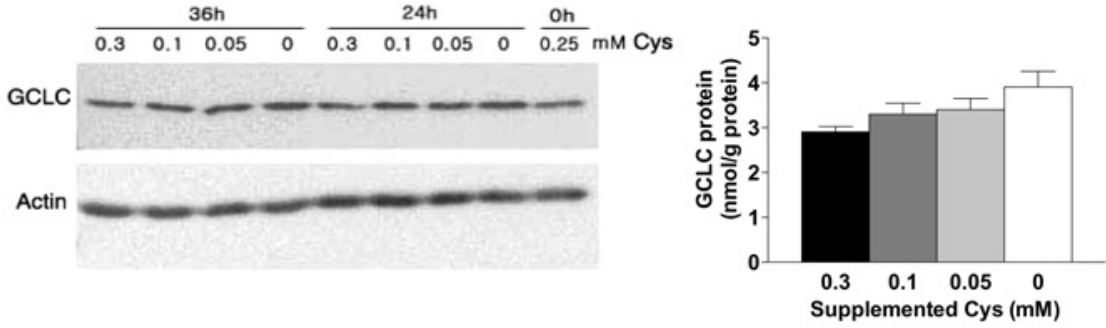

B
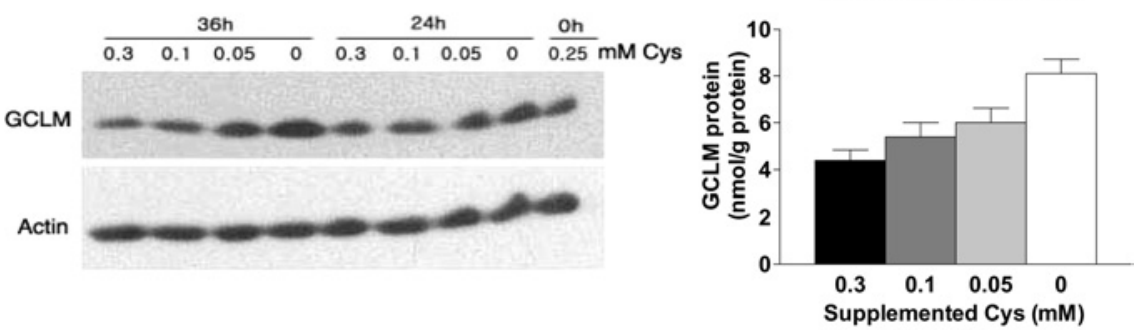

C
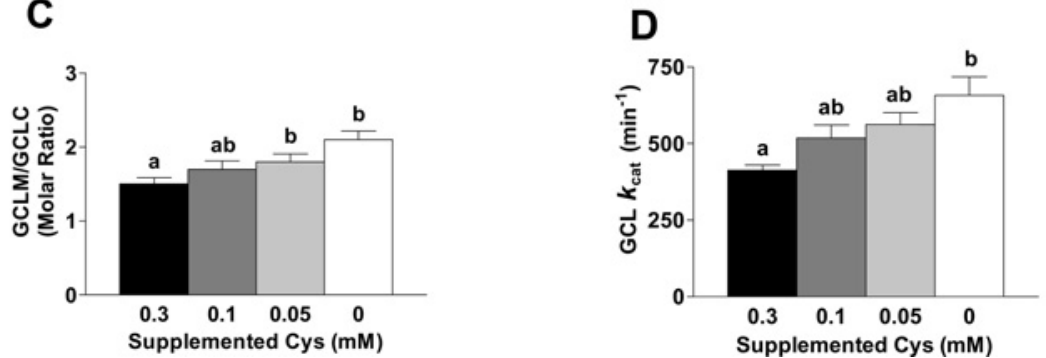

Figure 6 GCLC and GCLM levels, the GCLM to GCLC molar ratio and the $\boldsymbol{k}_{\text {cat }}$ of GCL are increased in response to cysteine deprivation in HepG2/C3A cells

(A) and (B) Western blot analysis of GCLC (A) and GCLM (B) in HepG2/C3A cells cultured in medium with $0.1 \mathrm{mM}$ methionine plus the designated concentration of cysteine for $36 \mathrm{~h}$. Equal amounts $(100 \mu \mathrm{g})$ of total soluble protein were loaded in each lane and separated by SDS/PAGE under reducing conditions. The amounts of GCLC and GCLM shown in the bar graphs to the right of the immunoblots were calculated using a standard curve run on each gel by loading various amounts of purified GCLC or GCLM protein. (C) Molar ratios of GCLM/GCLC. The molar concentrations of GCLC and GCLM were calculated by dividing the amount of GCLC $(\mathrm{mg})$ or GCLM $(\mathrm{mg})$ by the molecular mass of the protein; the molar amounts were used to calculate the ratio. (D) The $k_{\text {cat }}$ values for GCLC in HepG2/C3A cells. The $k_{\text {cat }}$ was calculated by dividing the $V_{\text {max }}$ (mol of product formed per min) by the molar amount of GCLC. Bars not denoted by the same superscript letter are significantly different $(P \leqslant 0.05)$ as determined by ANOVA and Tukey's $\omega$-procedure. Each value in bar graphs is the mean \pm S.E.M. for three replicates.

rats and cultured HepG2 cells. The up-regulation of GCL activity in response to cysteine deprivation is consistent with previously reported observations in rat liver and cultured rat hepatocytes [1517]. In the HepG2 cell system, we showed that regulation of GCL activity in response to cysteine level was dose-dependent, as has previously been reported for GCL activity in rat liver [17].

By quantitatively measuring the amount of GCLC protein, we have now been able to demonstrate for the first time that in both the liver of intact rats and HepG2 cells, changes in the GCLC level plus changes in the $k_{\text {cat }}$ of GCL entirely account for changes in GCL activity in response to cysteine availability. The fact that this regulation occurs in vivo over a range of protein levels that encompasses the requirement level suggests that this is a physiologically significant mechanism for regulation of GCL activity.

\section{Increases in the $\boldsymbol{k}_{\text {cat }}$ of GCL are associated with increases in the GCLM/GCLC molar ratio}

The levels of both GCLC and GCLM increased in response to cysteine deprivation in both rat liver and HepG2 cells, but there was a greater increase in the level of GCLM than in that of GCLC, resulting in an increase in the molar ratio of GCLM/GCLC. The observed subunit molar ratios were $0.2-0.3$ for GCL in the liver of intact rats and 1.5-2.1 for GCL in HepG2 cells. Thus the increase in the $k_{\text {cat }}$ was associated with an increase in the GCLM/GCLC molar ratio both when GCLM was limiting for holoenzyme formation and when it was present in excess of the molar level of GCLC. This seems to indicate that a large excess of GCLM is necessary to maintain GCLC in holoenzyme form.

Rat liver GCLC migrated largely as the GCLC monomer after non-reducing SDS/PAGE, consistent with the low GCLM/GCLC ratio in rat liver. Mouse liver, similar to rat liver, contains a very low ratio of GCLM/GCLC [27], but Yang et al. [7] found that mouse GCLM eluted only in the holoenzyme fraction after gel filtration chromatography. Assuming that rat GCLM also exists primarily as the GCLC-GCLM heterodimer in rat liver in vivo and that the $k_{\text {cat }}$ of rat GCL holoenzyme is 12 times that of the GCLC monomer, a $10 \%$ increase in the GCLM/GCLC ratio would increase the apparent $k_{\text {cat }}\left(V_{\max }\right)$ by $120 \%$, which is what we observed in the liver of rats fed a low-protein diet.

\section{Increases in the $\boldsymbol{k}_{\text {cat }}$ for GCL and in the GCLM/GCLC molar ratio are associated with increases in stable holoenzyme formation}

Although only approx. $20 \%$ of GCLC in rat liver was present as holoenzyme, most of the GCLC was present as holoenzyme in HepG2 cells. This is consistent with the higher molar ratio of GCLM/GCLC in HepG2 cells. The proportion of GCLC resolving as holoenzyme versus monomer after non-reducing 
A

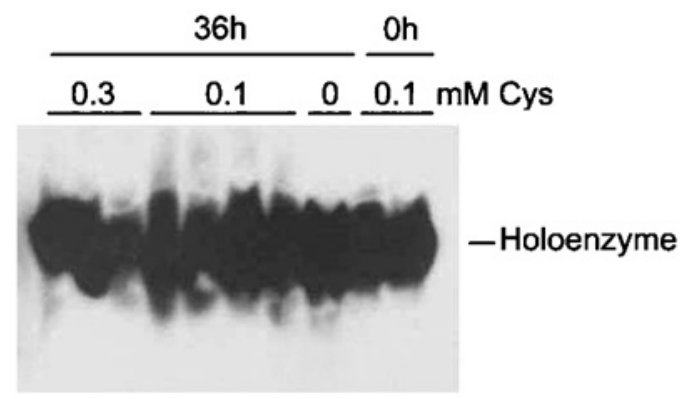

B

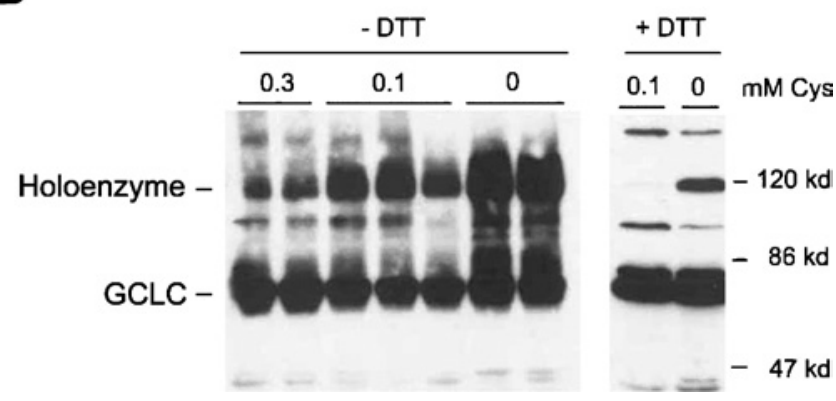

Figure 7 Increases in the GCLM/GCLC molar ratio and $\boldsymbol{k}_{\text {cat }}$ of GCL are associated with holoenzyme formation in HepG2/C3A cells

HepG2/C3A cells were cultured in medium with $0.1 \mathrm{mM}$ methionine and the designated concentration of cysteine for $36 \mathrm{~h}$. Equal amounts $(100 \mu \mathrm{g})$ of total soluble protein from the cultured cells were subjected to native PAGE (A) or SDS/PAGE (B). Membranes were blotted with anti-GCLC to detect holoenzyme and GCLC monomer. (B) The blot on the left is for samples run under non-reducing conditions (no DTT, no heat) and the blot on the right is for samples that were heated in $80 \mathrm{mM}$ DTT for 4 min at $95^{\circ} \mathrm{C}$ before SDS/PAGE.

SDS/PAGE was greater for HepG2 cells cultured with less cysteine, suggesting that the strength of holoenzyme association was greater for cells cultured in low-cysteine medium. Furthermore, a portion of the holoenzyme in cells that had been cultured in the no-cysteine-supplemented medium was resistant to separation of subunits even under reducing and denaturing conditions. These observations suggest that the degree or strength of association of the GCL subunits may vary for holoenzyme formed under different intracellular conditions and that different types of association of subunits may play a role in modulating the activity of the catalytic subunit.

Consistent with this suggestion, the addition of recombinant GCLM to rat liver homogenates was associated with a change from approx. $20 \%$ of GCLC in holoenzyme form in untreated rat liver homogenate to approx. $80 \%$ in holoenzyme form when excess GCLM was added. The increase in the GCLM/GCLC ratio in homogenates was accompanied by a $41 \%$ increase in GCL activity. The increase in activity observed in vitro is less than what would have been predicted based on observed changes in GCL subunit ratios and GCL activity in rat liver or HepG2 cells. Although human GCLM is very similar to rat GCLM, it is possible that the use of recombinant His $_{6}$-tagged human GCLM may have affected the association of the modifier subunit with rat GCLC, due either to species differences or to the presence of the $\mathrm{N}$ terminal histidine residues. Effects on subunit association could affect the kinetic parameters of the holoenzyme. Alternatively, the nature of the associations formed under in vitro incubation conditions may differ from those formed in vivo. In any case, the holoenzyme formed in vitro did not exhibit as great an increase in the $k_{\text {cat }}$ as would have been predicted by our results in rat liver and HepG2 cells.

It has frequently been stated, based on the report of Huang et al. [5], that the $V_{\max }$ of rat GCLC does not increase with holoenzyme formation. Our results for rat liver GCL suggest otherwise, i.e. that holoenzyme formation is associated with relatively large changes (i.e. 12-fold) in the $V_{\max }$ or $k_{\text {cat }}$ of rat GCLC. Studies of human GCL and Drosophila GCL have yielded $k_{\text {cat }}$ values that were 3-8× as high for GCL holoenzyme as for the GCLC subunit alone [2830]. A lack of adequate cellular cysteine appears to be associated with an increase in the extent of GCL holoenzyme formation and with an increase in the strength of association of subunits, and both of these appear to be associated with increases in the $k_{\text {cat }}$. Because cysteine is normally the limiting substrate for GSH synthesis and the $K_{\mathrm{m}}$ for cysteine is not affected by the presence of GCLM or holoenzyme formation [6,7,29], an increased $k_{\text {cat }}$ should translate to increased GSH synthesis.

The magnitude of the effect that GCLM expression can potentially have on GSH production is demonstrated by the recent characterization of the $G C L M^{-/-}$mouse. Despite a compensatory increase in GCLC, tissue levels of GSH in the $\mathrm{GCLM}^{-/-}$mice were only $9-16 \%$ of those in wild-type littermates and $43-82 \%$ of those in heterozygote littermates [7]. Transient transfection of COS-1 cells with the GCLM cDNA caused an increase in GCL activity of approx. 2-fold and an increase in GSH levels from $127 \pm 4$ to $179 \pm 19 \mathrm{nmol} / \mathrm{mg}$ of protein (mean \pm S.E.M.) [31]. These results are consistent with our suggestion that an increase in the GCLM/GCLC ratio will have a large effect on GCL activity and hence GSH synthesis. Similarly, the lack of saturation of GCLC with GCLM in liver in vivo allows efficient regulation of GCL activity by modulation of GCLM expression.

It is possible that an altered thiol/disulphide redox environment resulting from cysteine and GSH depletion might induce stronger bonds between the two subunits of GCL as a mechanism for increasing the GCL holoenzyme activity under conditions of cysteine deficiency. Using GCL from Drosophila mellanogaster, Fraser et al. [28] showed that association of GCLC with mutant GCLM (with three cysteine residues mutated to glycine residues) doubled the $k_{\text {cat }}$ of the catalytic subunit, whereas its association with wild-type GCLM increased the $k_{\text {cat }}$ to $8 \times$ that of GCLC alone. They hypothesized that some of the mutated cysteine residues were responsible for disulphide bond formation between the subunits. Additionally, Seelig et al. [32] reported that 10$25 \%$ of GCL holoenzyme purified from rat kidney remained after incubation for $24 \mathrm{~h}$ at $4{ }^{\circ} \mathrm{C}$ in the presence of $260 \mathrm{mM} \mathrm{DTT}$, and Tu and Anders [33] reported that purified human GCL holoenzyme was not completely dissociated into GCLC and GCLM subunits after incubation with $50 \mathrm{mM}$ DTT. These observations suggest that covalent interactions can form between the two subunits.

\section{Differential regulation of GCLM and GCLC levels}

It has previously been thought that GCLC and GCLM are regulated in a similar manner such that GCLC would be present predominantly as holoenzyme in most tissues. However, our results indicate that GCLC exists mainly as the GCLC monomer, with at most $20-30 \%$ as GCL holoenzyme in rat liver. Disproportionate regulation of the two subunits can thus play a role in the regulation of GCL activity. Our results indicate that an increase in GCLM level would have a much greater effect on GCL activity and GSH production in vivo than would an equimolar increase in GCLC level. 
This is one of the few studies in which the actual molar ratio of GCLC and GCLM subunits has been measured. Krzywanski et al. [27] reported that GCLM to GCLC ratios ranged between 0.06 and 0.33 in HBE1 (human bronchial epithelial 1) cells cultured under control conditions and were $0.10,0.25$ and 1.0 for mouse liver, kidney and heart respectively. The ratios we observed in rat liver are consistent with those found in mouse tissues and in HBE1 cells. However, the ratios we observed in HepG2/ C3A cells, with more GCLM than GCLC, appear not to be representative of the ratios present in hepatocytes in vivo.

In contrast with our observation that the GCLM/GCLC ratio consistently increased under conditions of cysteine limitation, Krzywanski et al. [27] consistently observed decreases in the GCLM/GCLC ratio in HBE1 cells treated with 4-hydroxy-2nonenal, 2,3-dimethoxy-1,4-naphthoquinone, or t-butylhydroquinone as models of oxidative stress that are known to work through different mechanisms. The opposite responses of the GCLM/GCLC ratio to cysteine deprivation and to models of oxidative stress supports the hypothesis that regulation of GCLM expression and of the GCLM/GCLC ratio is a major mechanism for regulation of GCL activity in response to cysteine availability, whereas up-regulation of GCLC transcription may be the major mechanism by which the cell responds to oxidative or chemical stress. Because we observed regulation of GCL activity by GCLM in the liver of rats fed on diets with protein intakes that ranged from $50 \%$ to $200 \%$ of the daily requirement level, it seems likely that regulation of GCL activity by an increase in the GCLM level is an important regulatory mechanism under normal physiological conditions. Formation of the holoenzyme would increase the $K_{\mathrm{i}}$ for GSH and increase the $k_{\text {cat }}$ of the enzyme, both of which should enhance GSH synthesis by GCL under physiological conditions.

\section{Mechanism of regulation of GCLC and GCLM levels}

Although there are many reports of increases in the levels of mRNAs for GCLC and GCLM in response to oxidative stress, only a few studies have addressed the relationship between mRNA abundance and the level of the translated protein or the potential mechanisms of post-transcriptional regulation $[27,33,34]$. In the present study, we found that increases in the mRNAs for GCLC and GCLM in rat liver were accompanied by parallel changes in GCLC and GCLM subunit levels, whereas the pattern and degree of changes in mRNA levels in HepG2 cells did not correlate as well with the gradual and step-wise changes in GCLC and GCLM protein levels. It is possible that GCLC and GCLM are partially regulated by changes in mRNA translational efficiency or by changes in protein degradation in the HepG2 cells.

A much broader range of cysteine and GSH levels were obtained in the studies with HepG2 cells than were seen in the liver of rats fed on diets with protein contents that were below or above the daily requirement level. Despite the insignificant effect of cysteine or methionine level in the medium on the level of ROS, as measured by DCF production, the changes in intracellular GSH concentration imply a greater oxidized thiol/disulphide redox balance in HepG2 cells deprived of cysteine than in the liver of rats fed on the low-protein diet. Electrophile response elementmediated up-regulation of GCLC and GCLM gene expression may have occurred in the cysteine-depleted HepG2 cells despite a lack of evidence for increased ROS production.

Nevertheless, it seems clear that levels of both GCLC and GCLM closely parallel the levels of GCLC and GCLM mRNAs in vivo and that changes in GCL activity in rat liver, as well as in HepG2 cells, are due to the additive effects of changes in the amount of GCLC and the $k_{\text {cat }}$ of GCLC. Our results also indicate that the $k_{\text {cat }}$ of GCLC is increased by holoenzyme formation.
Furthermore, our results indicate that the GCLM level in rat liver is limiting and that up-regulation of the GCLM level can result in increased holoenzyme formation and a potentially large increase in the $k_{\text {cat }}$. The lack of saturation of GCLC with GCLM appears to underline the cell's ability to efficiently regulate GCL activity and maintain GSH homoeostasis in the face of sulphur amino acid limitation.

We thank Larry L. Hirschberger for technical support. Supported by National Institutes of Health grant PHS DK 0664303 to M. H.S.

\section{REFERENCES}

1 Dickinson, D. A., Levonen, A. L., Moellering, D. R., Arnold, E. K., Zhang, H., Darley-Usmar, V. M. and Forman, H. J. (2004) Human glutamate cysteine ligase gene regulation through the electrophile response element. Free Radical Biol. Med. 37, 1152-1159

2 Lu, S. C. and Huang, C. S. (1994) Comparison of sulfur amino acid utilization for GSH synthesis between HepG2 cells and cultured rat hepatocytes. Biochem. Pharmacol. 47, 859-869

3 Gomi, A., Masuzawa, T., Ishikawa, T. and Kuo, M. T. (1997) Posttranscriptional regulation of MRP/GS-X pump and $\gamma$-glutamylcysteine synthetase expression by 1(4-amino-2methyl-5-pyrimidinyl)-methyl-3-(2-chloroethyl)-3-nitrosourea and by cycloheximide in human glioma cells. Biochem. Biophys. Res. Commun. 239, 51-56

4 Sekhar, K. R., Long, M., Long, J., Xu, Z. Q., Summar, M. L. and Freeman, M. L. (1997) Alteration of transcriptional and post-transcriptional expression of $\gamma$-glutamylcysteine synthetase by diethyl malate. Radiat. Res. 147, 592-597

5 Huang, C. S., Anderson, M. E. and Meister, A. (1993) Amino acid sequence and function of the light subunit of rat kidney $\gamma$-glutamylcysteine synthetase. J. Biol. Chem. 268, 20578-20583

6 Huang, C. S., Chang, L. S., Anderson, M. E. and Meister, A. (1993) Catalytic and regulatory properties of the heavy subunit of rat kidney $\gamma$-glutamylcysteine synthetase. J. Biol. Chem. 268, 19675-19680

7 Yang, Y. M., Dieter, Z., Chen, Y., Shertzer, H. G., Nebert, D. W. and Dalton, T. P. (2002) Initial characterization of the glutamate-cysteine ligase modifier subunit Gclm (-/-) knockout mouse. J. Biol. Chem. 277, 49446-49452

8 Sekura, R. and Meister, A. (1977) $\gamma$-Glutamylcysteine synthetase. Further purification, 'half of the sites' reactivity, subunits, and specificity. J. Biol. Chem. 252, 2599-2605

9 Richman, P. G. and Meister, A. (1975) Regulation of $\gamma$-glutamylcysteine synthetase by nonallosteric feedback inhibition by glutathione. J. Biol. Chem. 250, 1422-1426

10 Anderson, M. E. (1998) Glutathione: an overview of biosynthesis and modulation. Chem.-Biol. Interact. 111, 1-14

11 Griffith, 0. W. (1999) Biologic and pharmacologic regulation of mammalian glutathione synthesis. Free Radical Biol. Med. 27, 922-935

12 Kwon, Y. H. (1998) Regulation of cysteine dioxygenase and $\gamma$-glutamylcysteine synthetase in response to sulfur amino acids in primary cultures of rat hepatocytes. Doctoral dissertation, Cornell University Library, Ithaca, NY, U.S.A.

13 Bella, D. L., Hirschberger, L. L., Hosokawa, Y. and Stipanuk, M. H. (1999) Mechanisms involved in the regulation of key enzymes of cysteine metabolism in rat liver in vivo. Am. J. Physiol. 276, E326-E335

14 Bella, D. L., Hahn, C. and Stipanuk, M. H. (1999) Effects of nonsulfur and sulfur amino acids on the regulation of hepatic enzymes of cysteine metabolism. Am. J. Physiol. 277, E144-E153

15 Kwon, Y. H. and Stipanuk, M. H. (2001) Cysteine regulates expression of cysteine dioxygenase and $\gamma$-glutamylcysteine synthetase in cultured rat hepatocytes. Am. J. Physiol. Endocrinol. Metab. 280, E804-E815

16 Lee, J.-I., Londono, M. and Stipanuk, M. H. (2004) Regulation of cysteine dioxygenase and $\gamma$-glutamylcysteine synthetase is associated with hepatic cysteine level. J. Nutr. Biochem. 15, 112-122

17 Stipanuk, M. H., Londono, M., Lee, J.-I., Hu, M. and Yu, A. F. (2002) Enzymes and metabolites of cysteine metabolism in nonhepatic tissues of rats show little response to changes in dietary protein or sulfur amino acid levels. J. Nutr. 132, 3369-3378

18 Cresenzi, C. L., Lee, J.-I. and Stipanuk, M. H. (2003) Cysteine is the metabolic signal responsible for dietary regulation of hepatic cysteine dioxygenase and glutamate cysteine ligase in intact rats. J. Nutr. 133, 2697-2702

19 Yan, C. C. and Huxtable, R. J. (1996) Effects of monocrotaline, a pyrrolizidine alkaloid, on glutathione metabolism in the rat. Biochem. Pharmacol. 51, 375-379

20 Fariss, M. W. and Reed, D. J. (1987) High-performance liquid chromatography of thiols and disulfides: dinitrophemol derivatives. Methods Enzymol. 143, 101-109 
21 Stipanuk, M. H., Bagley, P. J., Coloso, R. W. and Banks, M. F. (1992) Metabolism of cysteine to taurine by rat hepatocytes. Adv. Exp. Med. Biol. 315, 413-421

22 Smith, P. K., Krohn, R. I., Hermanson, G. T., Mallia, A. K., Gartner, F. H., Provenzano, M. D., Fujimoto, E. K., Goeke, N. M., Olson, B. J. and Klenk, D. C. (1985) Measurement of protein using bicinchoninic acid. Anal. Biochem. 150, 76-85

23 Kikugawa, K., Yasuhara, Y., Ando, K., Koyama, K., Hiramoto, K. and Suzuki, M. (2003) Effect of supplementation of $n-3$ polyunsaturated fatty acids on oxidative stress-induced DNA damage of rat hepatocytes. Biol. Pharm. Bull. 26, 1239-1244

24 Cai, Z., Huang, Z. Z. and Lu, S. C. (1997) Differential regulation of $\gamma$-glutamylcysteine synthetase heavy and light subunit gene expression. Biochem. J. 326, 167-172

25 Toroser, D. and Sohal, R. S. (2005) Kinetic characteristics of native gamma-glutamylcysteine ligase in the aging housefly, Musca domestica $L$. Biochem. Biophys. Res. Commun. 326, 586-593

26 Gipp, J. J., Bailey, H. H. and Mulcahy, R. T. (1995) Cloning and sequencing of the cDNA for the light subunit of human liver gamma-glutamylcysteine synthetase and relative mRNA levels for heavy and light subunits in human normal tissues. Biochem. Biophys. Res. Commun. 206, 584-589

27 Krzywanski, D. M., Dickinson, D. A., Iles, K. E., Wigley, A. F., Franklin, C. C., Liu, R. M. Kavanagh, T. J. and Forman, H. J. (2004) Variable regulation of glutamate cysteine ligase subunit proteins affects glutathione biosynthesis in response to oxidative stress. Arch. Biochem. Biophys. 423, 116-125

Received 11 July 2005/25 August 2005; accepted 2 September 2005

Published as BJ Immediate Publication 2 September 2005, doi:10.1042/BJ20051111
28 Fraser, J. A., Kanasagra, P., Kotecki, C., Saunders, R. D. C. and McLellan, L. I. (2003) The modifier subunit of Drosophila glutamate-cysteine ligase regulates catalytic activity by covalent and noncovalent interaction and influences glutathione homeostasis in vivo. J. Biol. Chem. 278, 46369-46377

29 Fraser, J. A., Saunders, R. D. C. and McLellan, L. I. (2002) Drosophila melanogaster glutamate-cysteine ligase activity is regulated by a modifier subunit with a mechanism of action similar to that of the mammalian form. J. Biol. Chem. 277, 1158-1165

30 Tu, Z. and Anders, M. W. (1998) Identification of an important cysteine residue in human glutamate-cysteine ligase catalytic subunit by site-directed mutagenesis. Arch. Biochem. Biophys. 354, 247-254

31 Tipnis, S. R., Blake, D. G., Shepherd, A. G. and McLellan, L. I. (1999) Overexpression of the regulatory subunit of $\gamma$-glutamylcysteine synthetase in HeLa cells increases $\gamma$-glutamylcysteine synthetase activity and confers drug resistance. Biochem. J. 337, 559-566

32 Seelig, G. F., Simondsen, R. P. and Meister, A. (1984) Reversible dissociation of $\gamma$-glutamylcysteine synthetase into two subunits. J. Biol. Chem. 259, 9345-9347

33 Liu, R. M., Gao, L., Choi, J. and Forman, H. J. (1998) $\gamma$-Glutamylcysteine synthetase: mRNA stabilization and independent subunit transcription by 4-hydroxy2-nonenal. Am. J. Physiol. 275, L861-L869

34 Soltaninassab, S. R., Sekhar, K. R., Meredith, M. J. and Freeman, M. L. (2000) Multifaceted regulation of $\gamma$-glutamylcysteine synthetase. J. Cell. Physiol. 182, 163-170 